\title{
Ethics
}

\section{A Crisis in Chronic Pain Care: An Ethical Analysis. Part Three: Toward an Integrative, Multi-disciplinary Pain Medicine Built Around the Needs of the Patient}

James Giordano, $\mathrm{PhD}^{1}$, and Michael E. Schatman, $\mathrm{PhD}^{2}$

From: ${ }^{1}$ Georgetown University, Washington, DC; Center for Neurotechnology Studies, Potomac Institute of Policy

Studies, Arlington VA; and ${ }^{2}$ College of Osteopathic Medicine, Yakima, WA.

Dr. Giordano is SamueliRockefeller Professor, Departments of Medicine and

Neurosciences, Scholar-in-

Residence, Center for Clinical Bioethics, Georgetown University Medical Center, Washington, DC; and Senior Fellow and Director,

Center for Neurotechnology Studies, Potomac Institute of Policy Studies, Arlington, VA.

Dr. Schatman is Assistant

Professor, Department of Family

Medicine, Pacific Northwest

University of Health Sciences, College of Osteopathic Medicine, Yakima, WA.

Address correspondence: Dr. James Giordano

Departments of Medicine and Neurosciences Georgetown University Medical Center 4000 Reservoir Rd, Bldg D, Rm 238

Washington, DC 20057

E-mail:jg353@georgetown.edu

Disclaimer: Funded by a grant from the L.S. Rockefeller Trust, and an American Academy of Pain Medicine-Pfizer National Visiting Professor Award (JG) Conflict of interest: None.

Free full manuscript: www.painphysicianjournal.com
A number of variables have contributed to the current crisis in chronic pain care and are affected by, and affect, the philosophies and politics that influence the socio-economic climate of the American healthcare system. Thus, we posit that managing the crisis in chronic pain care in the United States is contingent upon the development of a multi-focal healthcare paradigm that more thoroughly enables and fortifies research, its translation (in education and practice), and the implementation of, and support for, both the curative and healing approaches in medicine in general, and pain care specifically. These steps necessitate re-examination, if not revision of the health care system and its economics.

The ethical imperative to consider and prudently employ cutting-edge diagnostic and therapeutic technologies in pain medicine is obligatory. However, "supply side prudence" is of little value if "demand side accessibility" is lacking. Revisions to health insurance plans advocated by the in-coming administration seek to create uniformity in basic health care services based upon re-assessment of the clinical effectiveness (versus merely cost) of treatments, including those that are "high tech." These plans attempt to allow every patient a more complete ability to deliberatively work with physicians to access those services and resources that maximize health functioning and goals. But even given these revisions, authentic pain care must take into account the interactive contexts of the painient individual. The biopsychosocial model of chronic pain management may have significant practical and ethical worth in this regard. A system of pain treatment operating from a biopsychosocial perspective necessitates integrative multi-disciplinarity. We propose a tiered, multi-disciplinary paradigm based upon the differing needs of each specific patient. But establishing such a system does not guarantee access, and distribution of these services and resources requires economic support to ensure that capabilities are more broadly available (i.e., supplied), and afforded as needed and wanted (i.e., demanded).

Toward this end, we posit the need to focus upon, and more fully integrate 1) education, 2) multi-disciplinary care (including re-vivification of MPCs), 3) policies that allow financial subsidies that afford patients the latitude to access and utilize such expanded resources appropriately to meet identified medical needs, and 4) medico-legal initiatives and statutes that protect and enable patients and physicians. The proposed changes comport with a number of ethical systems in that they support the basic deontic structure of the profession and allow for a richer, more finely grained articulation of clinical and ethical responsibilities within the scope of particular general, specialty, and sub-specialty practices.

Key words: Pain medicine, ethics, policy, integrative care, multi-disciplinary pain care

Pain Physician 2008; 11:6:775-784 
Introduction: Problems as Object LESSONS

As discussed in the first 2 papers in this series $(1,2)$, a number of variables have contributed to the current crisis in chronic pain care. If we are to effect ethically and practically meaningful outcomes from this Decade of Pain Control and Research, it is vital to not merely identify the variables that have instigated this crisis, but to analyze how problems have occurred and might be corrected - on both a paradigmatic scale as relates to pain medicine as a profession, and on a more immediate level that translates into the distinct realms of its constituent general, specialty, and sub-specialty practices.

Of those factors that play a role in shaping the current inadequacies of pain care, first and foremost is the complexity of chronic pain itself. We must abandon those orientations that treat pain as an object independently of the being that it affects. Any construct of pain medicine must be built upon an evolving understanding of pain (as physiological and psychological event) and its manifestations in a painient subject. This mandates 1) a continuous investment in research to further elucidate mechanisms, effects, and the viability of various therapeutics; 2) ongoing revision of clinical methods to reflect the most current knowledge, and 3) progressive subsidy to allow state-of-the-science techniques and technologies to be broadly available to pain patients.

These points speak to the second major factor, namely that pain occurs in individuals that are embedded within the fabric of environment, society, and culture (3), and as such are directly affected by the philosophies and politics that influence and dictate the socio-economic climate of the healthcare system. On the whole, the current state of pain care in the United States has been profoundly affected by discrepancies in resource and service access, polyglot public values, market-based restrictions in the provision and insurance support of clinical services (4), and the litigious nature of pain practice (Table 1 ).

But while a call for change is evident, the more difficult, yet absolutely essential task at hand is to weigh the benefits and burdens of any proposed revisions relative to proximate and more distal consequences within the healthcare system and to society at large, and implement those changes that evoke the most saliently beneficial (viz., most therapeutically and morally sound) effect(s). In the main, managing the crisis in chronic pain care in the United States is contingent upon the development of a multi-focal healthcare paradigm that more thoroughly enables and fortifies research, its translation (in education and practice), and the implementation of, and support for both the curative and healing approaches in medicine in general, and pain care more specifically. However, these steps necessitate re-examination (if not revision) of the healthcare system and its economics, and our hope is that the problems of the past several years serve as object lesson(s) to conduce positive change in the future.

Table 1. Socio-economic and legal factors contributing to the current crisis in pain care in the US.

Social Factors

1. Discrepancies in access to pain care

2. Demographic distribution of pain specialists and sub-specialists

3. Hegemony within pain medicine

4. Omnipresence and omnipotence of market forces

5. Differences in individual, and community values, and ethics

Economic Factors

1. Relative/perceived cost(s) of high tech vs. low tech diagnostics and therapeutics

2. Rationing of diagnostic and treatment options by third party carriers

3. Market model restrictions in pain care services that promote demand escalation

4. Inequality and inquities in insurance provision and support
Administrative and Legal Issues

1. Restrictions in third party claims' allowances

2. Discrepancies regarding "reality" and verifiability of pain and its causes

3. Litigious nature of pain-related cases (e.g., compensation claims, etc.)

4. Professional liabilities related to practice of pain medicine (e.g., opioid prescribing, malpractice, etc.)

For a complete discussion of these issues and problems in pain medicine, see also: Giordano J, Schatman ME [1]. 


\section{The Value of "Low-" and "High-Tech" Approaches}

Edwards (5) has advocated "...the duty to do all that can be done within the limits of current medical knowledge and available resources to relieve...pain." We most assuredly agree; yet the question remains how best to achieve these ends. For sure, the development of new biomedical techniques and technologies is conceptually oriented toward enhancing the capabilities of medical care (6-8). Often the advances that are most readily considered for, and/or utilized in clinical therapeutics are actually a combination of existing and new technologies $(8,9)$. The price of such technologies is dictated, at least partly, by the extent and costs of time and effort(s) required for development, testing, and evaluation that are then superimposed upon the costs of the technical elements themselves. Thus, the categorization of particular approach as "low-" or "high- tech" frequently reflects costs of development, production, and cost-redemptive use, rather than the actual sophistication of the technique(s) or technologies, per se (10). Given Edwards' (5) invocation for maximum effort, the ethical imperative to employ cutting-edge diagnostic and therapeutic technologies in pain medicine almost becomes axiomatic. Yet, it must be remembered that the underlying moral imperative that dictates this use is the fundamental good that is achieved and afforded through the relief of pain. Thus, any and all such tools should not be used simply because they are new while excluding other, older, yet still clinically viable techniques. Rather, to uphold instrumental value toward achieving the overall good of pain medicine, we have urged careful consideration and prudence in determining how, why, and when any technique or technology can and should be used in practice $(11,12)$.

However, such "supply side prudence" is of little value if "demand side accessibility" is lacking once these "high tech" tools and practices are deemed reasonable, and are in place and ready to use. The availability and use of high-technologies is often the province of specialists and sub-specialists, and even within the scope of these practices, third party payers may disallow pre-certification, or refuse reimbursement of advanced diagnostic and therapeutic technologies (13). The proposed revisions to healthcare insurance plans advocated by Senator Obama would seek to create uniformity in basic level of healthcare services that is based upon re-assessment of the clinical effec- tiveness (versus merely cost) of treatments, including those that are new, innovative, and have higher fixedor variable costs of adoption or use, respectively (14). The universality of care offered by these plans does not infer that every service will be provided to every patient, but rather that every patient has the ability to access those services and resources that maximize health functioning and goals, as realistically determined through deliberative guidance provided by his/her physician (15). This would instill a higher value for patient agency (and autonomy, not as proactive choice, but as the negative right to refuse particular treatment(s) based upon relative values and goals), and fortify the importance of active informed consent as means to address benefits, burdens, and risks of treatments as relevant to patients bio-psychosocial situations $(16,17)$. These situations obviously differ along a number of lines, and often reflect the variable demographics and circumstances of healthcare in rural and/or urban settings.

A recent study has demonstrated that the United States faces a serious shortage of pain specialists, which is most pronounced in rural areas (18). Accordingly, chronic pain in rural areas is treated almost exclusively by primary care physicians. Although these providers may be aware of the empirical value of advanced technologies and novel techniques, they may not have access to such approaches and/or their patients may not have health insurance that reimburses these services (or any health insurance at all!). Similarly, there are large numbers of un-insured, underinsured, and indigent patients in urban areas. As a result, many physicians, in both rural and urban areas, may feel that they have limited clinical options, and this may force acquiescence to a "one nail, one hammer" approach that contributes to the over-use of some treatments (e.g., long-term and improper use of opioids, polypharmacy, certain interventional techniques and/or technologies) and under-use of others (i.e., both high- and low-tech) that are more clinically appropriate $(19,20)$.

Reinforcing this, Rollin Gallagher (21) has emphasized that "...the cognitive skills of pain medicine must be emphasized, not the procedural skills..." urging the need for "...careful evaluation of the patient's full scope of problems or the cost-effectiveness of treatment." It may be that a combination of low- and hightech approaches is more clinically (as well as time- and cost-) effective in ameliorating pain, and restoring functional agency to the patient, and thereby more 
ethically sound (in terms of meeting the telic rightness and goodness of medicine) than the use of either approach alone $(10,22)$.

\section{Biopsychosocial Orientation and InTEGRATIVE Focus}

But while we advocate the use of new and novel technologies, it is critical that each and all of these implements be utilized in ways that best accommodate the patient. Toward this end, any authentic form of pain care must take into account the interactive contexts and effects of the painient individual and his/ her environment(s) $(3,23)$. Undeniably, chronic pain is perpetuated by a combination of physical, psychological, and environmental (i.e- socio-cultural) factors $(3,23,24)$. The biopsychosocial model of chronic pain management is most closely associated with these domains and as such may have significant practical and ethical worth (25). In its "real" form (i.e., as devised and advocated by George Engel (26), versus more "new age" iterations), the ethical value is derived from an intellectual appreciation of the interaction of causes and effects, and the relation of these contingencies to the moral responsibility to 1 ) recognize the patient as a person consisting of embodied and interacting systems, and 2) develop and enact good care that reflects and acknowledges this understanding (27). This is of paramount importance, as Halstead (28) has illustrated that any progress in medicine will be impeded as long as parochial distinctions continue to be drawn between "scientific" (i.e., technologically based) healthcare that focuses upon curing disease and enhancing physiologic function, and "humanistic" healthcare that focuses on healing the person. We concur with Halstead, believe these distinctions to be arbitrary at best, and specious at worst, and therefore call for a new, more complementary paradigm for treating chronic pain that eschews such "either-or" distinctions, and instead seeks to utilize the most current techniques and technologies together with those (older and non-technical approaches) that have proven, sustained utility to provide both curative and healing dimensions of pain care, as needed (29-31). Such a system of pain treatment operating from a biopsychosocial perspective would necessitate an integrative multidisciplinarity, with clinical collaboration focused upon treating the person-in-pain, rather than merely pain-as-object.

As matter of fact, multidisciplinary pain care was somewhat de rigueur in the United States until the late 1990s (32), at which time restricted insurance funding resulted in the absorption of multidiscplinary pain centers (MPCs) into other hospital-based programs (e.g., general rehabilitation), or closure due to relative lack of profit versus operational and overhead cost(s). Thus, while there were once over 1,000 MPCs in the United States, the number has dwindled to approximately 200 over the past decade (32). The decrease in multidisciplinary pain management in the United States has occurred despite multiple meta-analyses and systematic reviews supporting the efficacy and cost-efficiency of this approach (33-39).

Of course, we recognize that all MPCs were not model programs - each had limitations and many have failed. In certain cases, these failures were due to excessive utilization and replication of tests and procedures, the use of a regimented, non-individualized approach to pain care, and/or a clinician employing allied health providers (or ancillary care providers such as massage therapists), and claiming such an enterprise to be a multidisciplinary treatment facility. Semantics aside, the simple inclusion of more than one clinician does not genuinely fulfill the professional obligation of what a "multidisciplinary pain center" purports to do or be, and these claims border on frank misrepresentation. Hence, the abundance of insurance claims for such bastardized forms of "multidisciplinary pain care" became difficult, if not impossible to substantiate, and in many ways this cupidity led to both the progressive decrement in insurance re-imbursement of any/all multidisciplinary pain care, and the implicit zero-sum competition that arose between clinics and single-provider forms of pain management.

This is not to infer that all MPCs were contributory to this trend toward self-demise. Many MPCs provided valid, sound integrative pain care, but the aforementioned financial strains that diminished reimbursement, coupled to other socio-economic factors that escalated medical costs, led to pain centers being absorbed into, or overtaken by, larger health service corporations, or disbanded altogether (40). While the number of multidisciplinary chronic pain management programs in the United States has decreased, other nations are steadily increasing the availability of multidisciplinary treatment $(41,42)$. Although efforts are being made to "revive" multidisciplinary chronic pain management in the United States, such an undertaking is likely to be an extremely slow process due to the current economic 
milieu, governmental, private insurance, and hospital administrative incentives for cost-containment, and a reluctance to re-incur past errors $(43,44)$. But if we are to learn from the past so as to effectively and ethically navigate this crisis, it becomes important to revise the infrastructure and social construct of pain medicine so as to be responsive to the needs of patients, and still be economically and administratively sustainable $(45,46)$.

Although Gatchel and colleagues (47) have demonstrated that multidisciplinary chronic pain programs are less effective when selected elements of the programs are "carved out" by insurance companies or the programs themselves (due to non-reimbursement of group services), we opine that multidisciplinary treatment for pain can still be achieved outside the context of a traditional comprehensive multidisciplinary program. Doing so, however, will be complicated as it involves a coordinated effort among general, specialty, sub-specialty, and allied health practitioners, and may require that insurance protocols be re-examined and restructured so that healthcare professionals can provide prolonged collaborative services for which they currently may not be reimbursed.

As attractive as such specialized, integrative, and high-tech pain care may be, in reality the majority of pain is addressed and managed in a primary care setting (despite the fact that limitations upon the scope of primary care often restrict the comprehensive treatment required to manage the chronic pain) (48). This does not reflect any distinct incapacity of primary care; rather, we believe that the market forces that have been imposed upon the regnant medical culture have disenabled primary care practice, and threatened the viability of truly integrative care of the patient with intractable pain (49).

Frequently, it is the primary care physician who first encounters the patient with durable and/or intractable chronic pain, and must therefore render a diagnosis that (initially) will determine the type, scope, and trajectory of subsequent care (whether it be rendered within primary care setting or afforded by specialists, sub-specialists, and/or MPCs) (50). Diagnosis and treatment of the chronic patient are difficult even for the well-trained pain specialist, and, as Gallagher (51) has noted, "...we have only a few thousand pain specialists...those often have fragmented training....and most primary pain care doctors have little focused training in pain management... ."

\section{A Unified Approach: Steps Toward Change}

In light of this, we maintain that the problem of chronic pain requires a more expansive paradigm of care that conjoins the primary care physician to act within a larger network of pain practitioners and MPCs, and that policy must be established that recognizes the epidemiologic, social, and personal burden of pain, and which facilitates a role for primary care in an integrative context of specialty, sub-specialty, and MPC-based pain medicine. Moreover, the economics of third party reimbursement within the current system of medical care does not enable the primary care physician to allocate the time or clinical efforts necessary to address chronic pain, nor do these economics sustain viable resources (such as referrals to specialists, sub-specialists, or MPCs) to provide the comprehensive care that is required. Medico-legal and administrative issues result from the escalating pressures to treat such patients within a context of diminishing opportunities for the provision of, and/or referral to, more appropriately expansive care (refer to Table 1). Thus, for a strengthened, integrative pain medicine to be developed, exist, and prosper, it must be empowered by economic and administrative support.

Obviously, this calls for a sea change. Toward this end, we propose system-wide revisions on (at least) 3 levels:

First, physicians must be more completely educated about pain. This must occur 1) during the didactic, basic sciences component of the medical school years as part of an expanded curriculum that incorporates not only the scientific understanding of pain, but a more humanistic appreciation of the nature and impact of suffering as a primary focus of medicine qua medicine; 2) during the clinical clerkship phase of medical education so as to expose medical students to the realities of pain and the possibilities of pain medicine; 3 ) during the residency experience of any/ all specialties that encounter pain patients within the scope of their practice(s), and 4) throughout clinicians' careers through the provision of ongoing continuing education that allows integration of high-level knowledge to even the most "grass-roots" medical practice, such that an understanding of the issues of pain, viabilities of low- and high-tech approaches to treating pain patients, validity and limitations of particular clinical practice(s), and networks for referral can be established (52). 
Second, efforts must be taken to revivify MPCs, and establish an infrastructure of pain medicine that facilitates referral to, and maintenance of, these treatment centers (49). The general mission of this network of pain medicine would be to provide continuity of pain care, using an integrative, multidisciplinary approach that accommodates a broader base of medical resources to provide an enhanced capacity for long-term treatment, management, and palliative care, rather than the fragmentary, single practitionerbased system as is currently being utilized. In this way, specialists, sub-specialists, and MPCs would provide expert knowledge to, and/or implement, pain management beyond the limitations imposed upon the primary care physician. Primary care physicians could elect to collaborate with pain specialists, sub-specialists, and the MPC to co-manage each patient. Such reciprocally informative and deliberative partnering between various physicians, clinicians of the MPC, and the patient would allow for the most erudite, patientfocused clinical equipoise, and would enable the provision of the best care. This multipartite relationship would also reduce 1) potential compliance issues (e.g, regarding pharmacologic use, dose escalation, and misuse/abuse), and 2) adversarial interactions involving third-party carriers (e.g. non-substantiation of reimbursement for medical services needed) and regula- tory agencies (e.g. legal sanctions) that have become increasingly intimidating, and which have contributed to under-treatment of pain via "defensive" medical practice. In this latter regard, it is frequently issues surrounding longitudinal management of the pain patient that constitute the basis for a defensive stance. Indeed, there are special challenges when treating the patient who presents with co-morbidities such as depression, anxiety, psychotic and personality disorders, substance abuse and/or addiction $(53,54)$. But rather than distancing such patients from the care they require, this networked model would afford resources for information (e.g. knowledge about spectrum effects, awareness of drug interactions, tolerance, etc), implement precautions (e.g. identification of potential liabilities, use of screening tools, etc.), provide patient-attribute-specific treatment (e.g. employing genomics and biomarkers to guide use of particular pharmacologic agents, techniques, and technologies), and monitoring/care by professional specialists (e.g. psychiatry/psychology, addiction medicine, etc.) to focus upon the needs of each patient, as required. The core objectives of this proposed system of comprehensive pain care are described in Table 2. The grounds and basis for these referrals (e.g. to specialists, subspecialists, and MPCs) reflect the complexity of pain, and the need for primary care to function coopera-

Table 2. Specific objectives of a comprehensive, integrative system of multidisciplinary pain care.

1. Continuity and enhancement of patients' pain care through collaboration(s) between primary care, specialty, and subspecialty physicians

2. Use of an integrative, multi-disciplinary approach with broadly based medical resources with increased capacity for treatment(s)

3. Provision of individualized pain care, based upon specific needs of each patient

4. Treatment that accommodates both curative and healing paradigms

5. Prudent use of, and access to both low- and high-tech diagnostic and therapeutic approaches

6. Provision, subsidy, and support of both outpatient and in-patient pain care

7. Outsourcing partnerships with mental health programs to address psychiatric factors and accommodate psychological dimensions of pain
8. Outreach social service and educational programs to accommodate social and economic factors

9. Establishment and subsidy of diagnostic and outcomes databases at the local, state and national levels

10. Facilitated cooperation with third party payers to support prudent clinician-guided and patient values-based utilization of techniques, technologies, and programs

Such a system of leveled, integrative multi-disciplinary pain care is based upon the moral obligation to 1) acknowledge pain as a complex, biopsychosocial spectrum disorder, and 2) recognize and respond to the need for low- and high-tech diagnostics and therapeutics that are focused upon both curative and healing dimensions of personalized, patient-centered care. To articulate this project requires legislation and policy that is directed at providing federal and privatized economic support for research, education and the constituent clinical practice(s) in order to maximize best use of available knowledge, technologies, and resources. (See text for detail.) 
tively with other medical specialties and disciplines to provide comprehensive, effective, and ethical treatment for each person in pain.

We propose a multilevel scheme of pain care, in which Level I management would and should be administered within the primary care setting. Level II care would be dedicated to those specific pain conditions that require specialty (i.e. pain physician) and/or sub-specialty treatment(s) for ongoing management (e.g. neurology, rheumatology, etc.), and Level III care would be trans-disciplinary, focusing upon pain as illness, and would be the purview of the MPC.

Third, such programs must be supported, developed, and maintained in ways that reciprocally allow for 1) financial subsidies that afford patients the latitude to appropriately access and utilize such expanded resources to meet their identified medical need(s), and 2) development of a system of medicolegal initiatives and statutes that protect and enable patients and physicians within a re-established probity of the physician-patient relationship; thereby assuaging patients' fears of inaccessibility to care, and physicians' fears that they will be unable to render such care because of economic, administrative, and/or legal restrictions. But here too, it is best to proceed with caution, as policy that merely acknowledges the impact of pain, but does not generate economic support for programs that are specifically designed and implemented for achieving positive therapeutic outcomes might only serve to worsen social discord (Table 1).

\section{Integrative Pain Care: A Proposed Articulation}

In this series of papers, we have attempted to depict the complex nature of pain, ethical complications and difficulties that arise in the treatment of the pain patient, a putative ethical framework for pain medicine, and the need for a re-integration of primary, specialty, sub-specialty and multidisciplinary care within a larger context of pain care. Toward this latter goal we offer the following suggestions:

1. The primary care physician should provide, and be qualified (by dedication, education, training, and experience) to guide Level I management of the pain patient.

2. Referrals to specialists, sub-specialists, and MPCs by the primary care physician should reflect the prudent judgment that more advanced consultation, if not treatment, is required.

3. Policy should be in place to insure that patients re- turn to the primary care physician (i.e. Level I care) following Level II or III consultation or required treatment, unless otherwise indicated.

4. The primary care physician can elect to participate with specialists, sub-specialists, and clinicians of the MPC in providing continuity of care to the extent that the primary care physician feels competent and/or amenable.

5. Level III MPC care can be provided in collaboration with referring Level I and/or II clinicians, as needed and/or desired, and represents the appropriate type and extent of care required based upon the type, severity, and/or co-morbidity of pain and other conditions.

We feel that our proposal cannot be static, but must reflect a cycle of understanding and progress. Collaboration should be developed between primary care providers, specialists, sub-specialists, and MPCs that is guided by patient and community needs, and fostered by local cooperation. Referrals by primary care physicians, and the treatment options that are available and provided by specialists, sub-specialists, and within the MPC should be individualized. Multidisciplinary pain centers should also contribute to research databanks that seek to inform and guide clinical diagnostics and therapeutic outcomes. As new information is gained from these outcomes' findings, and other areas of research, it must be incorporated into education and translated into clinical practice(s) (55-57). Systems and policies for the furtherance of such research and the delivery of progressively advanced care must be equally fluid, and must reflect not only utilization of healthcare resources in pain medicine in ways that are right, but the sound dedication to treating pain in ways that fulfill healthcare's role as a public good.

\section{Ethical Value}

These steps uphold the moral obligation to effectively and efficiently treat pain, and comport with a number of ethical systems that describe and direct how such moral obligations might (and should) be enacted. We have claimed that "...the profession (of pain medicine) must be informed by scientific knowledge that is contemporary and progressive, but it must also be sensitive to the subjectivity of suffering, ...to apply knowledge and skill ... that ideally meets each patient's individual medical needs...this is the basis of medicine as tekne... that combines...skill and....art and which is integrative ... in the ideal (30)." In this way, pain care is consistent with, and adherent to 
the epistemic, anthropologic, and ethical domains of a core philosophy of medicine. Given that historicity and canon sustain any philosophy, the utility of philosophical content requires balancing the practical value and application(s) of old(er) and new(er) concepts so as to be most contemporarily relevant. This balance, as an operational construct of a philosophy of medicine upon which integrative pain care is built, would therefore compel obtaining, and prudently employing "...distinct and interactive forms of knowledge... in ways that ... become...open to the importance of, and requirements for distinct forms, types, and perspectives of therapeutic care" (30).

The proposed paradigm of integrative pain care 1) supports the basic deontic structure of the profession, 2) allows for a more complete articulation of clinical and ethical responsibilities within the scope of particular general, specialty, and sub-specialty practices, and 3 ) upholds the value of pain care as an interpersonal interaction that seeks to execute good acts and ends as specifically defined by the needs of the patient, and parameters of the clinical relationship $(2,58)$. The participatory rules define the general framework of pain medicine as a profession, and reinforce the role of the clinician as a moral agent. Such agency obligates 1) the most current understanding of pain, 2) familiarity with new techniques and technologies of pain care (59) and 3) the ability to intuit how, why, and when to use certain techniques and technologies. It becomes evident that the use of various diagnostic and therapeutic options is not only based upon technical knowledge, but also reflects clinicians' moral compass, and use of ethical frameworks and systems to sustain and justify the decisions made in particular cases.

It may be that certain medical specialties tend to operate in general accordance with a given ethical orientation as "befits" the focus and specifics of the practice. As well, particular individuals might be intellectually and/or emotionally drawn to certain specialties because of this ethical "flavoring" as aligned with their own moral values. We do not discount these possibilities, however, is more likely that a number of ethical systems are viable (and operational) within each specialty, and therefore an integrative, multidisciplinary approach would allow for a richer, discursively ethical approach to caring for pain patients, both individually, and as a population.

\section{From Philosophy to Policy}

In serving that population, pain medicine enacts a public good; therefore it must be accessible to those patients who require the benefit(s) it tenders. The proposed professional infrastructure makes available tiered, multidisciplinary care based upon the differing needs of each specific patient. But availability does not guarantee access, and the distribution of these services and resources requires healthcare policies that provide economic support to ensure that these capabilities are more broadly available (i.e., supplied), and accessed as needed and wanted (i.e., demanded).

Both clinicians and patients contribute to this demand, and there is incumbent responsibility upon clinicians to weigh the merit of low- and high-tech approaches as relative to the type of condition(s), and bio-psychosocial status of patient(s) treated so as to justify the supply of these resources and services to respective patient populations. Patient demand(s) for various high-tech services may be dictated and constrained by a number of bio-socio-economic factors, and even in a progressive economic scheme of healthcare benefits that is responsive to patient choice, the clinician must 1) be aware of the interplay of constraining factors that affect each patient, and 2) deliberate with individual patients to guide their demands for acquiring various diagnostic and therapeutic options - not as fiscal gatekeeper, but as a morally responsible steward of knowledge. On an individual level this requires collaboration not only with patients, but with other healthcare professionals, case managers, insurance providers, and hospital administrators to ensure that treatment guidelines exist and are maintained that allow access to, and use of, various lowand high-tech approaches. However, on a public level, guidelines and practices (if not the profession of pain medicine at-large) must be supported by policy.

The relationships between facts and realities, goals and ends, rules of profession and the therapeutic and ethical instantiations of those rules in the various practices of pain medicine have been addressed in the present series of papers, and elsewhere $(1,2,12,52,60$, 61). The structure and functions of all of these components are reciprocal: philosophy prompts science and science informs philosophy. Old and new philosophical concepts must be weighed for merit and utility in 
light of durable and changing factors within the scientific and humanistic domains in which philosophy is applied; old(er) and new(er) techniques and technologies must be balanced on worth, and both scientific and humanitarian orientations must be entailed in the articulation of medicine as a practice. Ultimately, the profession and practice are directed by guidelines, but both must be supported by policies and economics that enable the core philosophy to be enacted, and in so doing promote, advance, and sustain ethical good. In light of this, we believe that clinicians must play a strongly contributory role in guideline and policy formation, by working together with researchers, patient groups, hospital and insurance administrators, and law makers. As a new administration comes to the fore, let us hope that it is sensitive to these efforts, heeds the problems identified and resolutions proposed, and assumes a strong role in catalyzing positive change.

\section{Acknowledgments}

This work was adapted from a chapter titled: "Ethics in practice: Framework for an integrative multidisciplinary pain medicine" (Ethik in der Praxis: Grundlage für ein integrativer, Vielfachsdisziplinarschmerzmedizin), from the author's (JG) Sammel dissertation "Can the Neuroscience of Pain Inform and Sustain a Neurophilosophy and Neuroethics of Pain Care" (Von Nervenswissenschaft der Schmerz, bis ein Neurophilosophie und Neuroethik für Schmerzpflege) submitted in partial fulfillment of the requirements for the DPhil degree, Humboldt Universität, Berlin, Germany.

The authors thank Dr. Pierre LeRoy for intellectual contribution, and Holly Long and Sherry Loveless for editorial assistance. This work was supported by funding from the Laurance S. Rockefeller Trust, and an American Academy of Pain Medicine-Pfizer National Visiting Professorship (JG).

\section{References}

1. Giordano J, Schatman ME. A crisis in pain care: An ethical analysis. Part 1. Facts, issues, and problems. Pain Physician 2008; 11:483-490.

2. Giordano J, Schatman ME. A crisis in chronic pain care: An ethical analysis; Part 2. Proposed structure and function of an ethics of pain medicine. Pain Physician 2008; 11:589-595.

3. Morris D. The Culture of Pain., University of California Press, Berkeley 1991.

4. Giordano J, Hutchison PJ, Benedikter R. Regrounding medicine to a core philosophy amidst technocracy and a post modern mindset. J Soc Phil In press.

5. Edwards RB. Pain and the ethics of pain management. Soc Sci Med 1984; 18:515-523.

6. Rose S. The Future of the Brain: The Promise and Perils of Tomorrow's Neuroscience. , Oxford University Press, Oxford 2005.

7. Smith MR. Technological determinism in American culture. In: Smith M.R., Marx L. (eds.) Does Technology Drive History?: The Dilemma of Technological Determinism. MIT Press, Cambridge, 2001, p. 1-36.

8. Borgmann A. Technology and the Character of Contemporary Life: A Philosophical Inquiry. University of Chicago Press, Chicago, 1984.

9. Benford G, Malartre E. Beyond Human: Living with Robots and Cyborgs. Tom
Doherty Associates, NY, 2007.

10. McClellan M, Kessler D. A global analysis of technological change in health care: The case of heart attacks. Health Affairs 1999; 18:250-253

11. Giordano J. Technology in pain medicine: Research, practice, and the influence of the market. Prac Pain Management 2008; 8:56-59.

12. Giordano J. Techniques, technology and tekne: On the ethical use of guidelines in the practice of interventional pain medicine. Pain Physician 2007; 10:1-5.

13. Aspden P. (ed). Medical Innovation in the Changing Healthcare Marketplace. Institute of Medicine, Washington, DC, 2002.

14. Obama B. Candidate Commentaries. Modern Healthcare 2007; November 26; 12-21.

15. Satz AB. Toward solving the health care crisis: The paradoxical case for universal access to high technology. Yale J Health Policy, Law, and Ethics 2008; 23. 8:93-143.

16. Fulford KWM. Facts/Values: Ten principles of values-based medicine. In: Rad- 24 den J. (ed). The Philosophy of Psychiatry: A Companion. Oxford University Press, Oxford, 2004, p.205-236.

17. Waters D, Sierpina VS. Goal-directed health care and the chronic pain patient: 25 a new vision of the healing encounter. Pain Physician 2006; 9:353-360.
18. Breuer B. The epidemiology of pain. In: Pappagallo M. (ed). The Neurological Basis of Pain. McGraw-Hill, NY, 2005, pp.179-193.

19. Giordano J. Pain, the patient and the physician: Philosophy and virtue ethics in pain medicine. In: Schatman M. (ed). Ethical Issue in Chronic Pain Management. Taylor-Francis, NY, 2006, pp.1-18.

20. Yoshida GM, Nelson RW, Capen DA, Nagelberg S, Thomas JC, Rimoldi RL, Haye W. Evaluation of continuous intraspinal narcotic analgesia for chronic pain from benign causes. Am J Orthop 1996; 25:693-694.

1. Gallagher RM. Primary care and pain medicine: A community solution to the public health problem of chronic pain. Med Clin North Am 1999; 83:555-583.

22. Delbridge L., Robinson B. Genetic and biochemical screening for endocrine disease: III. Costs and logistics. World J. Surgery 1998: 22:121.

23. Giordano J.Complementarity, brainmind, and pain. Forsch Komplementarmed 2008; 15:2-6.

. Giordano J, Engebretson J, Benedikter R. Pain and culture: Considerations for meaning and context. Cambridge Quarterly Rev Healthcare Ethics 2008; 77: 45-59.

- Gatchel R, Peng Y, Peters M, Fuchs P, Turk D. The biopsychosocial approach to chronic pain: Scientific advances and 
future directions. Psychological Bulletin 2007; 133:581-624.

26. Engel GL. The need for a new medical model: A challenge for biomedicine. Science 1978; 196:129-136.

27. Ghaemi SN. The Concepts of Psychiatry: A Pluralistic Approach to the Mind and Mental Illness. Johns Hopkins University Press, Baltimore 2003.

28. Halstead LS. The John Stanley Coulter lecture. The power of compassion and caring in rehabilitation healing. Arch Phys Med Rehabil 2001; 82:149-154.

29. Giordano J. Maldynia: Pain as illness and the need for complmentarity in pain care. Forsch Komplementarmed 2008; 15 , in press.

30. Giordano J, Jonas WJ. Asclepius and Hygieia in dialectic: Philosophical, ethical and pragmatic bases of an integrative medicine. Integrative Med Insights 2007; 2:89-101.

31. Welie JVM. In the Face of Suffering: The Philosophical-Anthropological Foundations of Clinical Ethics. Creighton University Press, Omaha, 1998.

32. Meldrum ML. Brief history of multidisciplinary management of chronic pain, 1900-2000. In: Schatman MS, Campbell A. (eds.) Chronic Pain Management: Guidelines for Multidisciplinary Program Development. Informa Healthcare, New York, 2007:1-14.

33. Guzman J, Esmail R, Karjalainen L, Malmivaara A, Irvin E, Bombardier C. Multidisciplinary bio-psycho-social rehabilitation for chronic low back pain. Cochrane Database Syst Rev, 2002; 1 : CDooog63.

34. Hatzakis M, Schatman ME. The impact of interventional approaches when used within the context of multidisciplinary chronic pain management. In: Schatman ME, Campbell A. (eds). Chronic Pain Management: Guidelines for Multidisciplinary Program Development. Informa Healthcare, New York, 2007: 101-115.

35. Flor H, Fydrich T, Turk DC. Efficacy of multidisciplinary pain treatment centers: A meta-analytic review. Pain 1992; 49:221-230.

36. Turk DC. Okifuji A. Treatment of chronic pain patients: clinical outcomes, costeffectiveness, and cost-benefits of multidisciplinary pain centers. Crit Rev Phy Rehab Med 1998; 10:181-208.

37. Turk DC. Clinical effectiveness and cost-effectiveness of treatments for patients with chronic pain. Clin J Pain, 2002; 18:355-365.

38. Hoffman BM, Papas RK, Chatkoff DK, Kerns RD. Meta-analysis of psychological interventions for chronic low back pain. Health Psychology 2007; 26:1-9.

39. Guzman J, Esmail R, Karjalainen L, Malmivaara A, Irvin E, Bombardier C. Multidisciplinary rehabilitation for chronic low back pain : A systematic review. Br Medic / 2001; 322:1511-1516.

40. Schatman ME. The demise of multidisciplinary pain management clinics? Pract Pain Management 2006; 6:30-41.

41. Okifuji A, Turk DC, Kalauoklani D. Clinical outcome and economic evaluation of multidisciplinary pain centers. In: Block AR, Kramer EF, Fernandez E (eds). Handbook of Pain Syndromes: Biopsychosocial Perspectives. Lawrence Erlbaum Associates, Mahwah, N, 1999; 77-97.

42. Robbins H, Gatchel RJ, Noe C, Gajraj N, Polatin P, Deschner M, Vakharia A, Adams L. A prospective one-year outcome study of interdisciplinary chronic pain management: compromising its efficacy by managed care policies. Anesth Analg 2003; 97:156-162.

43. Hatten AL, Gatchel RJ, Polatin PB, Stowell AW. A cost-utility analysis of chronic spinal pain treatment outcomes: Converting SF-36 data into quality-adjusted life years. Clin J Pain 2006; 22:700-711.

44. Turk DC, Swanson K. Efficacy and costeffectiveness treatment for chronic pain: an analysis and evidence-based synthesis. In: Schatman ME, Campbell A, eds. Chronic Pain Management: Guidelines for Multidisciplinary Program Development. Informa Healthcare, New York, 2007:15-38.

45. Lenk H. Progress, values, and responsibility. Soc Phil Tech 1997; 2:102-119.

46. Giordano J. Cassandra's curse: Interventional pain management, policy and preserving meaning against a market mentality. Pain Physician 2006; 9:167170.

47. Gatchel RJ, Noe CE, Gajraj NM, Vakharia AS, Polatin PB, Deschner MD, Pulliam C. The negative therapeutic impact on an interdisciplinary pain management program of insurance "treatment carveout” practices. J Work Compens 2001; 10:50-63.

48. Garber A, Sox H. The U.S. physician workforce: serious questions raised, answers needed. Ann Int Med 2004; 141:732-734.

49. Giordano J. Pain medicine, morality and the marketplace; Time for a change. Prac Pain Management 2006; 6:88-89.

50. Gallagher RM. Primary care and pain medicine: A community solution to the public health problem of pain. Med Clin North America 1999; 83:555-583.

51. Gallagher RM. Congressional briefing on pain: HR 1020- the National Pain Care Policy Act. Pain Matters 2006; 10:2-5.

52. Maricich Y, Giordano J. Pain, suffering and the ethics of pain medicine: Is a deontic foundation sufficient? Am J Pain Management 2007; 17:130-138.

53. Kirsh K, Whitcomb LA, Donoghy K, Passik SD. Abuse and addiction issues in medically ill patients with pain: Attempts at clarification of terms and empirical study. Clin J Pain 2002; 18:S5260.

54. Manchikanti L, Giordano J, Fellows B, Manchukonda R, Pampati V. Role of psychological factors as predictors of opioid abuse and illicit drug use in chronic pain patients. J Opioid Management 2007; 3:89-101.

55. Giordano J. Pain research: Can paradigmatic revision bridge the needs of medicine, scientific philosophy and ethics? Pain Physician 2004; 7:459-463.

56. Giordano J. Good as gold? The randomized controlled trial: Pragmatic and ethical issues in pain research. Am J Pain Management 2006; 16;68-71.

57. Giordano J, Pedroni J. Pain research: The relationship of knowing and doing. Prac Pain Management 2007; 7:28-30.

58. Pellegrino ED. The healing relationship: Architectonics of clinical medicine. In: Shelp EE (ed). The Clinical Encounter: The Moral Fabric of the Physician-Patient Relationship. Reidel, Boston, 1983.

59. Giordano J. On knowing: Domains of knowledge and intellectual virtue in practical pain management. Prac Pain Management 2006; 6:65-76.

6o. Giordano J. Pain as disease and illness: Part 2 - Structure and function of the ethics of pain medicine. Prac Pain Management 2006; 6:65-68.

61. Giordano J. Grappling with the ethics of practical pain management. Prac Pain Management 2008; 8:72-75. 